\title{
Mathematical Model of Stock Prices via a Fractional Brownian Motion Model with Adaptive Parameters
}

\author{
Tidarut Areerak \\ School of Mathematics, Institute of Science, Suranaree University of Technology, Nakhon Ratchasima 30000, Thailand \\ Correspondence should be addressed to Tidarut Areerak; tidarut@sut.ac.th
}

Received 13 February 2014; Accepted 30 March 2014; Published 7 April 2014

Academic Editors: F. Sartoretto and C. Zhang

Copyright (C) 2014 Tidarut Areerak. This is an open access article distributed under the Creative Commons Attribution License, which permits unrestricted use, distribution, and reproduction in any medium, provided the original work is properly cited.

\begin{abstract}
The paper presents a mathematical model of stock prices using a fractional Brownian motion model with adaptive parameters (FBMAP). The accuracy index of the proposed model is compared with the Brownian motion model with adaptive parameters (BMAP). The parameters in both models are adapted at any time. The ADVANC Info Service Public Company Limited (ADVANC) and Land and Houses Public Company Limited (LH) closed prices are concerned in the paper. The Brownian motion model with adaptive parameters (BMAP) and fractional Brownian motion model with adaptive parameters (FBMAP) are applied to identify ADVANC and LH closed prices. The simulation results show that the FBMAP is more suitable for forecasting the ADVANC and LH closed price than the BMAP.
\end{abstract}

\section{Introduction}

The ideas of using a Brownian motion process to explain the behavior of the risky asset prices were presented by Black et al. [1-3]. The stock prices presented in the paper are also the type in the risky asset prices. Therefore, the Brownian motion is usually used to model a stock price. However, Brownian motion process has the independent increments property. This means that the present price must not affect the future price. In fact, the present stock price may influence the price at some time in the future. Hence, Brownian motion process is not suitable to explain the stock price. Another process, a fractional Brownian motion process, exhibits a long range dependent property. Therefore, a fractional Brownian motion process can be used to describe the behavior of stock price instead of Brownian motion process.

The rate of return and volatility in general asset pricing model are usually the constant parameters. Actually, the rate of return and volatility in the model are not constant at any time. In the paper, these parameters are updated depending on time by using the new information.

The ADVANC Info Service Public Company Limited (ADVANC) and Land and Houses Public Company Limited (LH) stock prices are considered in the paper. These two stocks are chosen from different stock exchange of Thailand
(SET) industry groups. The ADVANC and LH prices are selected from technology group (TECH) and property and construction group (PROPCON), respectively.

The ADVANC and LH stock price models are studied by using fractional Brownian motion process to explain uncertainly behavior instead of Brownian motion process. The Brownian motion model with adaptive parameters (BMAP) and the fractional Brownian motion model with adaptive parameters (FBMAP) are presented to model the ADVANC and LH stock prices.

The paper is organized as follows. Preliminaries on a fractional Brownian motion are given in Section 2. The estimation of the rate of return and volatility is shown in Section 3. In Section 4, the BMAP and the FBMAP are explained. Finally, Section 5 concludes the work in the paper.

\section{Preliminaries on a Fractional Brownian Motion Process}

In the general accepted model, the randomness of stock price is modelled by Brownian motion process. A stock price process $\left(S_{t}, t \geq 0\right)$ is represented by the stochastic differential equation (SDE) as shown in

$$
d S_{t}=S_{t}\left(\mu d t+\sigma d W_{t}\right) .
$$


Note that the parameters $\mu$ and $\sigma$ are the rate of return and the volatility, respectively. The process $\left(W_{t}, t \geq 0\right)$ in (1) is a standard Brownian motion process. The stochastic differential equation (1) is driven by the Brownian motion process $\left(W_{t}, t \geq 0\right)$. In the real world, $\mu$ and $\sigma$ in (1) are not constant at any time. Hence these parameters in the paper are the adaptable parameters based on time. In the paper, model (1) is called a Brownian motion model with adaptive parameters (BMAP).

In practice, the dynamics of stock price have a long memory (long range dependence). The BMAP model in (1) is not suitable to describe the dynamics of stock price. Therefore, the fractional Brownian motion process is considered in the paper. The fractional Brownian motion process $\left(B_{t}^{H}, t \geq 0\right)$ with Hurst index $H$ is a centered Gaussian process. If $H=0.5$, then $\left(B_{t}^{H}, t \geq 0\right)$ is a standard Brownian motion process. If $H \neq 0.5$, then $\left(B_{t}^{H}, t \geq 0\right)$ is neither a semimartingale nor a Markov process. For $H \neq 0.5$ case, the $\left(B_{t}^{H}, t \geq 0\right)$ is the long memory process. The $\left(B_{t}^{H}, t \geq 0\right)$ is represented in (2) by Mandelbrot and Van Ness [4]. Consider the following:

$$
B_{t}^{H}=\frac{1}{\Gamma(1+\alpha)}\left[Z_{t}+B_{t}\right] \text {. }
$$

The function $\Gamma(\cdot)$ is the gamma function. The process $\left(Z_{t}, t \geq\right.$ $0)$ is defined by $Z_{t}=\int_{-\infty}^{0}\left[(t-s)^{\alpha}-(-s)^{\alpha}\right] d W_{s}$. The process $\left(B_{t}, t \geq 0\right)$ is described by $B_{t}=\int_{0}^{t}(t-s)^{\alpha} d W_{s}$. The parameter $\alpha=H-1 / 2$, where $H \in(0,1)$ and $\left(W_{t}, t \geq 0\right)$ is a standard Brownian motion process.

The rate of return and volatility are not constant at any time. Hence, the paper also proposes the new approach of the asset pricing model. In this case, the driving process of model (1) is replaced by a fractional Brownian motion process. The rate of return and volatility are adaptive parameters. In this case, the model can be represented by the stochastic differential equation (SDE) as shown in

$$
d S_{t}=S_{t}\left(\mu d t+\sigma d B_{t}^{H}\right)
$$

The parameters $\mu$ and $\sigma$ in (3) are the rate of return and the volatility, respectively. The $\mu$ and $\sigma$ are adaptive parameters the same as the previous model. The $\left(B_{t}^{H}, t \geq 0\right)$ is a fractional Brownian motion process. In the paper, model (3) is called a fractional Brownian motion model with adaptive parameters (FBMAP).

Alòs et al. [5] have proposed to use the process $\left(B_{t}, t \geq\right.$ $0)$ instead of $\left(B_{t}^{H}, t \geq 0\right)$, since $\left(Z_{t}, t \geq 0\right)$ has absolutely continuous trajectory. So the process $\left(B_{t}, t \geq 0\right)$ has long range dependence. Hence, the model (3) can be considered as shown in

$$
d S_{t}=S_{t}\left(\mu d t+\sigma d B_{t}\right)
$$

An approximate approach to stochastic differential equation perturbed by fractional Brownian motion was proposed by Thao [6]. The process $\left(B_{t}^{\varepsilon}, t \geq 0\right)$ is introduced. For every $\varepsilon>0$, the process $\left(B_{t}^{\varepsilon}, t \geq 0\right)$ is defined by

$$
B_{t}^{\varepsilon}=\int_{0}^{t}(t-s+\varepsilon)^{\alpha} d W_{s} .
$$

The process $\left(B_{t}^{\varepsilon}, t \geq 0\right)$ is a semimartingale. Therefore, this process can be written as in

$$
B_{t}^{\varepsilon}=\alpha \int_{0}^{t} \varphi_{s}^{\varepsilon} d s+\varepsilon^{\alpha} W_{t},
$$

where $\varphi_{t}^{\varepsilon}=\int_{0}^{t}(t-s+\varepsilon)^{\alpha-1} d W_{s}$.

The process $\left(B_{t}^{\varepsilon}, t \geq 0\right)$ converges to $\left(B_{t}, t \geq 0\right)$ in $L^{2}(\Omega)$ when $\varepsilon$ approaches to 0 . This convergence is uniform with respect to $t \in[0, T]$. Hence, the model (4) can be considered as shown in

$$
d S_{t}=S_{t}\left(\mu d t+\sigma d B_{t}^{\varepsilon}\right)
$$

\section{The Estimation of the Rate of Return and Volatility}

In this paper, the ADVANC and $\mathrm{LH}$ closed prices are identified by two asset pricing models. In the BMAP, the driving process is Brownian motion. On the other hand, the driving process is fractional Brownian motion in the FBMAP. The parameters $\mu$ and $\sigma$ in both models are adaptive parameters at any time. The ADVANC and LH simulated stock prices are compared with these empirical prices. The ADVANC and LH empirical prices can be obtained from http://www.set.or.th/th/index.html. The data of ADVANC and LH empirical prices from July 9, 2010, to July 8, 2013, are used in the paper. These data are divided into two joint sets for two purposes. The first set (July 9, 2010-July 5, 2013) is used to estimate the drift rate and volatility. The second set (July 8 , 2011-July 8, 2013) is used for model validations.

The rate of return and volatility contained in the BMAP and the FBMAP are adaptive parameters based on time. Therefore, these parameters are not constant. In this section, the rate of return and volatility of ADVANC and LH stock prices are estimated.

The ADVANC and LH closed prices from July 9, 2010, to July 5,2013 , are used to estimate $\mu_{j}$ and $\sigma_{j}$ by using (8) and (9), respectively [7]. Consider the following:

$$
\begin{gathered}
\mu_{j}=\frac{252}{M} \sum_{i=1}^{M} R_{i}, \\
\sigma_{j}=\sqrt{\frac{252}{M-1} \sum_{i=1}^{M}\left(R_{i}-\bar{R}\right)^{2}},
\end{gathered}
$$

where $R_{i}$ is the return of stock price which can be computed by $R_{i}=\left(S_{i+1}-S_{i}\right) / S_{i}, \bar{R}$ is the average of return $R_{i}$, and $M$ is the number of returns. The parameters $\mu_{j}$ and $\sigma_{j}$ are estimated by the set of data as shown in Figure 1. In this figure, the data from July 9, 2010, to July 8, 2011, are used to estimate the initial $\mu_{0}$ and $\sigma_{0}$. The data from July 9, 2010, to July 11, 2011, are used to estimate $\mu_{1}$ and $\sigma_{1}$. The stock market is closed on the weekend. Therefore, the closed prices on July 9, 2011, and July 10, 2011, are not available. The data from July 9, 2010, to July 12,2011 , are used to estimate $\mu_{2}$ and $\sigma_{2}$, and so on. Using the same procedure, the data from July 9, 2010, to July 5, 2013, are used to estimate $\mu_{482}$ and $\sigma_{482}$. 


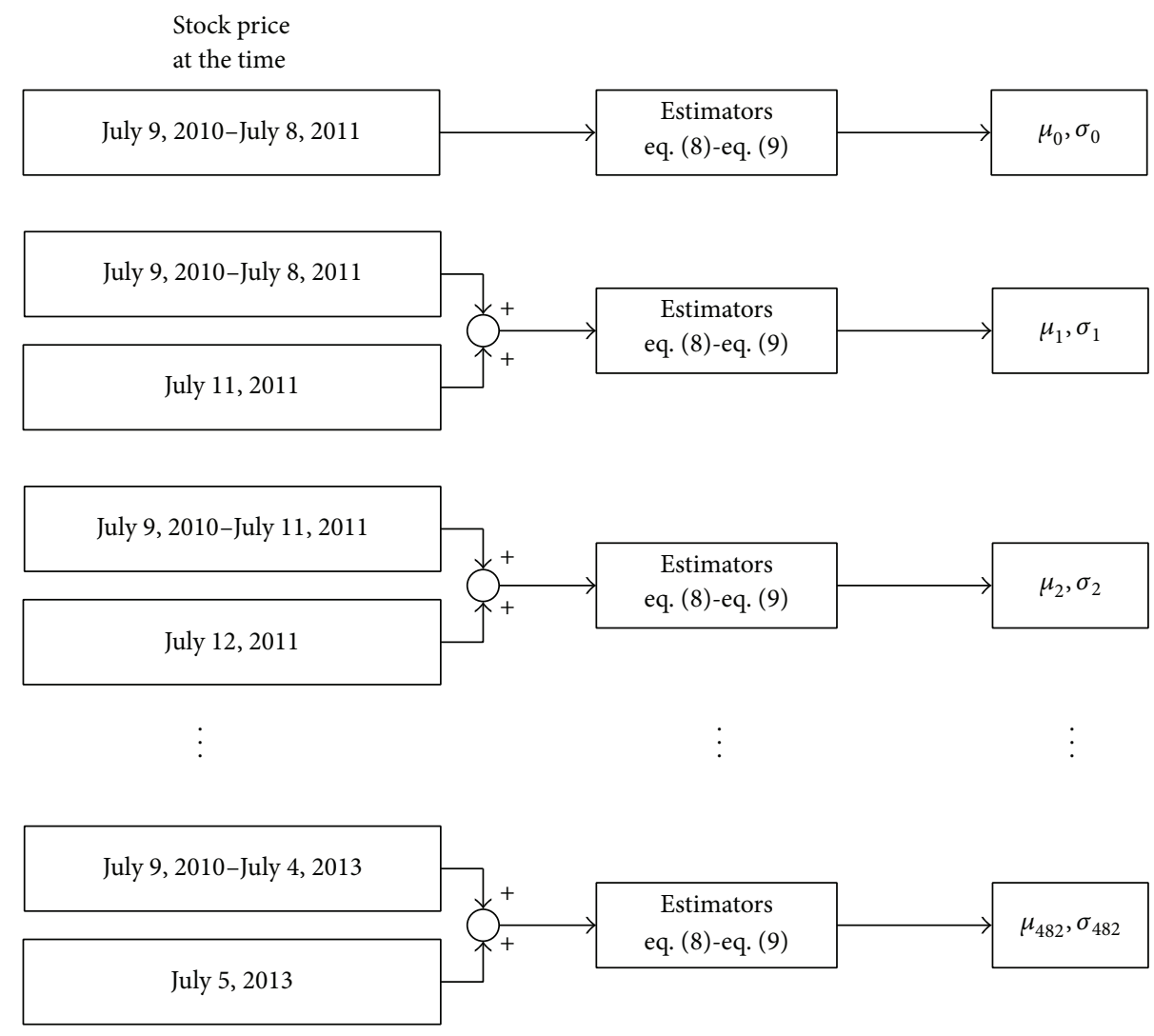

FIgURE 1: The flowchart to estimate the parameters $\mu_{j}$ and $\sigma_{j}$.

From the estimation results, the parameters $\mu_{j}$ and $\sigma_{j}$ of ADVANC and LH closed prices are shown in Figures 2 and 3 , respectively.

\section{Stock Prices Mathematical Models}

4.1. Brownian Motion Model with Adaptive Parameters (BMAP). The BMAP can be considered by the SDE as shown in (1). The rate of return $\mu$ and the volatility $\sigma$ are adaptive parameters and can be estimated using the flowchart in Figure 1. In the paper, the Euler discretization method is applied to solve the SDE. The solution of discretized form of the SDE (1) is denoted by $S_{j}$. Therefore, the Euler discretization form of (1) can be written in

$$
S_{j+1}=S_{j}+\mu_{j} S_{j} \Delta t+\sigma_{j} S_{j} \Delta W_{j},
$$

where $j$ is time index $(j=0, \ldots, N), N$ is the number of datasets, $\Delta t$ is a sampling time, and $\mu_{j}$ and $\sigma_{j}$ are estimated in the previous section. For the paper, $N$ is equal to 484 and $\Delta t$ is set to $1 / 252$. The initial value $S_{0}$ is equal to stock price at July 8,2011 . The term $\Delta W_{j}$ can be approximated by

$$
\Delta W_{j}=Z_{j} \sqrt{\Delta t}
$$

The random variable $Z_{j}$ is the standard normally distributed random variable with mean $=0$ and variance $=1$. It is generated by method of Box and Muller [8].
The ADVANC and LH stock prices calculated from the BMAP are simulated by MATLAB programming. These simulated data are compared with the second set data of empirical prices for a model validation. The average relative percentage error (ARPE) as given in (12) is the accuracy index in the paper. Consider the following:

$$
\mathrm{ARPE}=\frac{1}{N} \sum_{i=1}^{N} \frac{\left|X_{i}-Y_{i}\right|}{X_{i}} \times 100,
$$

where $N$ is the number of datasets, $X_{i}$ is the empirical price (market price), and $Y_{i}$ is the model price (simulated price).

For the simulation results by the BMAP, Figure 4 shows the empirical prices compared with the prices simulated by the BMAP for a given path of Brownian motion process. In the paper, the date period for simulation is between July 8 , 2011, and July 8, 2013.

\subsection{Fractional Brownian Motion Model with Adaptive Param-} eters (FBMAP). The FBMAP can be described by SDE (7). The rate of return $\mu$ and the volatility $\sigma$ in this model are variable parameters depending on time. The $\mu$ and $\sigma$ can be estimated using the block diagram as shown in Figure 1. The SDE (7) is solved by using the Euler discretization method. Therefore, the Euler discretization form of (7) can be written in

$$
S_{j+1}^{\varepsilon}=S_{j}^{\varepsilon}+\mu_{j} S_{j}^{\varepsilon} \Delta t+\sigma_{j} S_{j}^{\varepsilon}\left[\alpha \varphi_{j} \Delta t+\Delta W_{j} \varepsilon^{\alpha}\right] .
$$




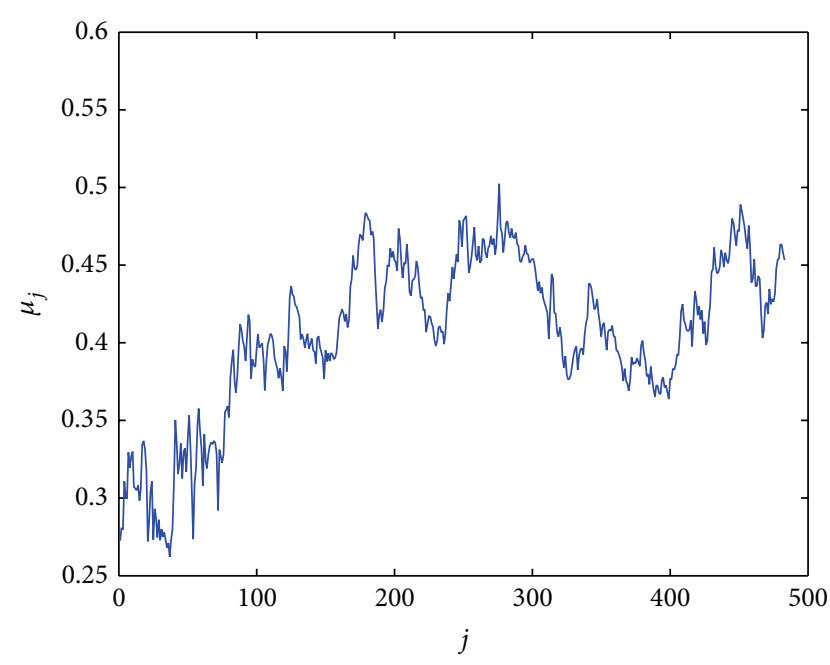

(a) ADVANC

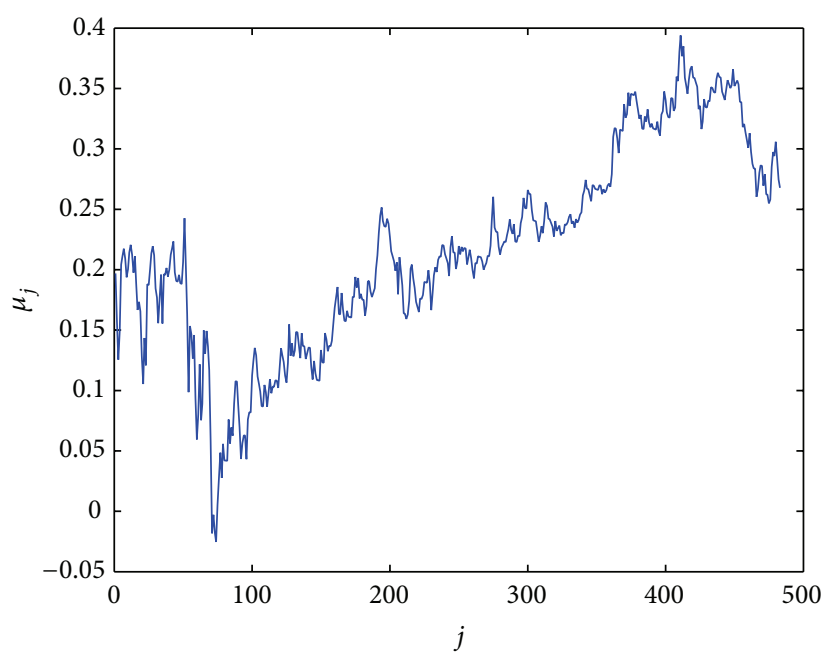

(b) $\mathrm{LH}$

FIGURE 2: The historical drift rate $\mu_{j}$ for closed prices prediction from July 11, 2011, to July 8, 2013.

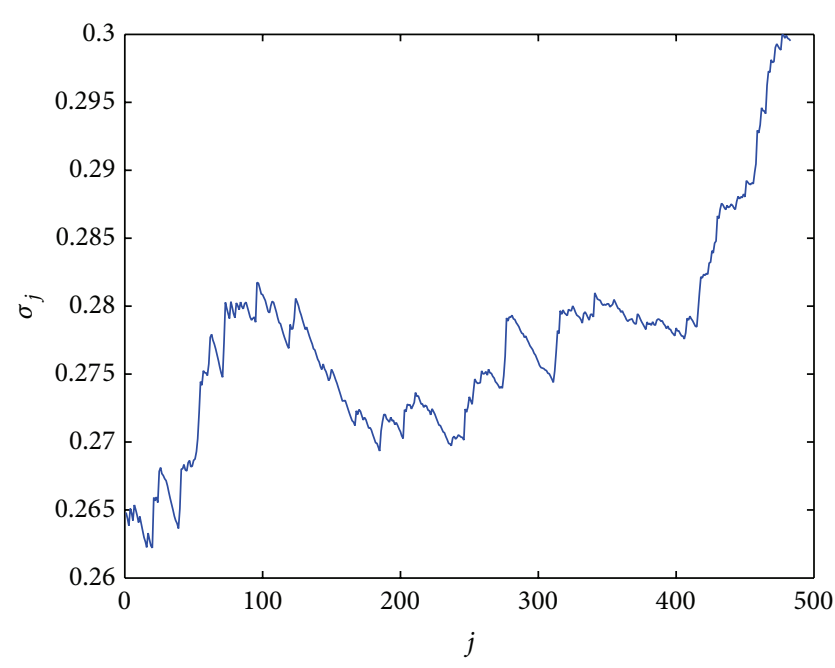

(a) ADVANC

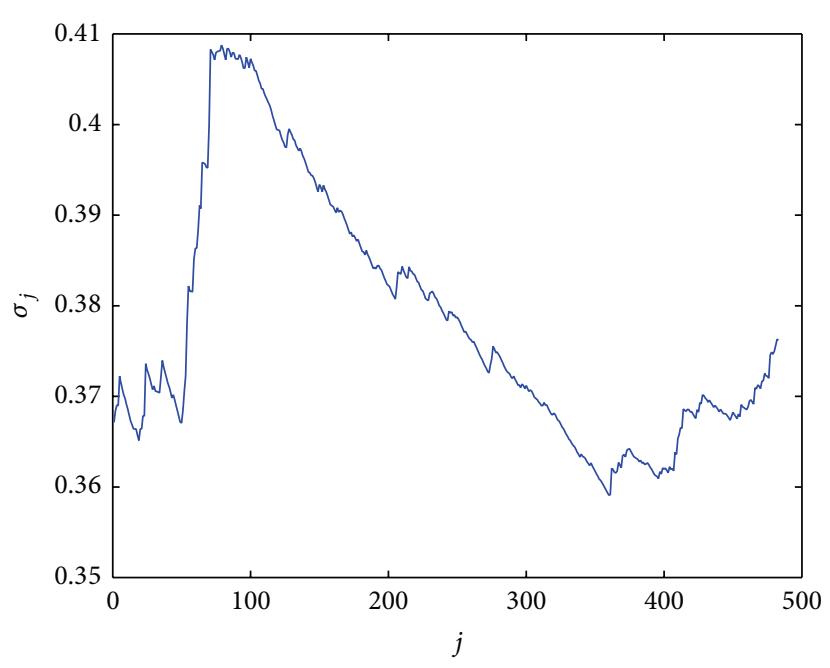

(b) $\mathrm{LH}$

FIGURE 3: The historical volatility $\sigma_{j}$ for closed prices prediction from July 11, 2011, to July 8, 2013.

In (13), the $S_{j}^{\varepsilon}$ is the discretized solution of the $\operatorname{SDE}(7), j$, $\Delta t$, and $N$ have the same meaning as the BMAP case. The parameter $\alpha=H-0.5$, where $H$ is Hurst index and $H \in(0,1)$. The estimation of this parameter is shown in Section 4.2.1. The parameters $\mu_{j}$ and $\sigma_{j}$ are calculated the same as those of the BMAP case. In the paper, $N, \Delta t$, and $\varepsilon$ are set equal to $484,0.005$, and $1 / 252$, respectively. The initial value $S_{0}^{\varepsilon}$ is equal to stock price at July 8,2011 . The term $\Delta W_{j}$ can be generated by (11). The term $\varphi_{j}$ in (13) can be calculated by [9]

$$
\varphi_{j}=\sqrt{\frac{j \Delta t}{N}} \sum_{k=0}^{N-1}\left(t-\frac{k j \Delta t}{N}+\varepsilon\right)^{\alpha-1} Z_{k}
$$

The random variable $Z_{k}$ in (14) is the standard normally distributed random variable with mean $=0$ and variance $=$ 1. It is generated by Box and Muller method.
The MATLAB programming is also used to calculate the ADVANC and LH closed prices in FBMAP. For model validation, these simulated data are compared with the empirical prices on July 8, 2011-July 8, 2013. The accuracy index in this case uses the average relative percentage error (ARPE) as calculated by (12).

4.2.1. Parameter Estimation. The parameters $\alpha$ for ADVANC and LH stock prices are the unknown values. Therefore, the estimation of these parameters is proposed in this section. The parameter $\alpha$ is calculated by $\alpha=H-0.5$. In this equation, $H$ is Hurst index and $0<H<1$. Hence $-0.5<\alpha<0.5$. Firstly, $\alpha$ is varied from -0.5 to 0.5 with step size equal to 0.1 . However, the parameter $\alpha$ cannot be equal to -0.5 or 0.5 . Therefore, -0.49 and 0.49 are used instead of -0.5 and 0.5 , respectively. The 10,000 sample paths of Brownian motion 


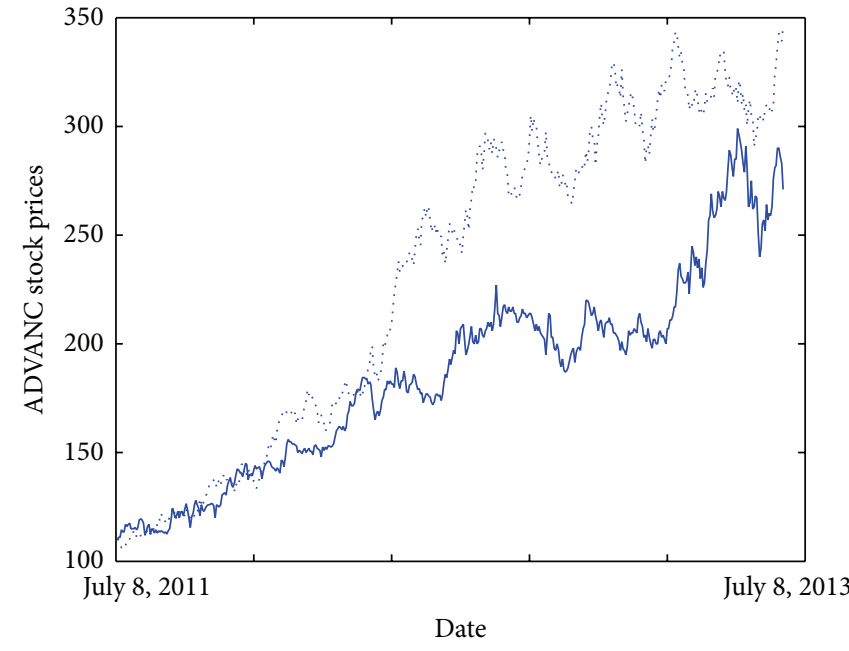

ADVANC empirical prices ADVANC prices simulated by BMAP

(a) For ADVANC closed prices $(\mathrm{ARPE}=23.268 \%)$

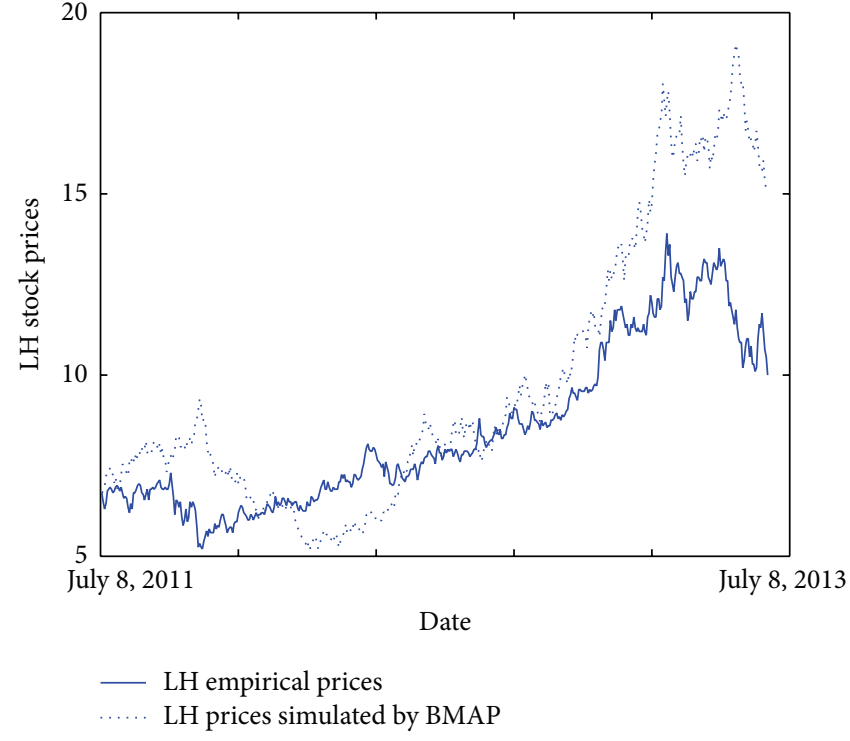

(b) For LH closed prices $(\mathrm{ARPE}=18.483 \%)$

FIGURE 4: The simulation results using the BMAP.

TABLE 1: ADVANC.

\begin{tabular}{|c|c|c|c|c|c|c|c|c|c|c|c|}
\hline $\bar{\alpha}$ & -0.49 & -0.4 & -0.3 & -0.2 & -0.1 & 0 & 0.1 & 0.2 & 0.3 & 0.4 & 0.49 \\
\hline Average of ARPE & 12.383 & 14.713 & 21.128 & 32.555 & 42.932 & 21.496 & 114.55 & $3.8315 \times 10^{14}$ & $3.7474 \times 10^{210}$ & Inf & Inf \\
\hline SD of ARPE & 3.5199 & 5.4755 & 9.3025 & 18.166 & 30.492 & 10.474 & 744.64 & $2.8687 \times 10^{16}$ & Inf & Inf & Inf \\
\hline
\end{tabular}

TABLE 2: LH.

\begin{tabular}{lccccccccccc}
\hline$\alpha$ & -0.49 & -0.4 & -0.3 & -0.2 & -0.1 & 0 & 0.1 & 0.2 & 0.3 & 0.4 & 0.49 \\
\hline Average of ARPE & 13.362 & 17.722 & 28.059 & 44.745 & 59.309 & 29.370 & 118.19 & $5.0349 \times 10^{41}$ & $8.0212 \times 10^{268}$ & Inf & Inf \\
SD of ARPE & 3.3415 & 6.2432 & 13.703 & 31.977 & 59.243 & 15.935 & 749.36 & $4.0179 \times 10^{43}$ & Inf & Inf & Inf \\
\hline
\end{tabular}

process are considered. In each path, the average relative percentage error (ARPE) is computed using every value $\alpha$. The simulation results for ADVANC and LH stock prices with $\alpha$ varied from -0.49 to 0.49 are addressed in Tables 1 and 2, respectively.

It can be seen that the average and standard deviation of ARPE in case of $\alpha=-0.49$ are the minimum in both stock prices (ADVANC and LH). Therefore, $\alpha=-0.49$ is chosen for ADVANC and LH cases.

4.2.2. Model Validation. For the simulation results using the FBMAP, Figure 5 shows the empirical prices compared with the prices simulated by the FBMAP (7) with the same scenario of Brownian motion of Figure 4. In Figure 5, the date period to simulate the stock prices using FBMAP is between July 8, 2011, and July 8, 2013.

4.3. Comparison of Accuracy Index between BMAP and FBMAP. For a given standard Brownian motion sample path, Figures 4 and 5 show that the average relative percentage
TABLE 3: The average and standard deviation of ARPE using the BMAP and FBMAP.

\begin{tabular}{lccc}
\hline Stock name & Model & $\begin{array}{c}\text { Average of } \\
\text { ARPE }\end{array}$ & $\begin{array}{c}\text { Standard deviation } \\
\text { of ARPE }\end{array}$ \\
\hline ADVANC & BMAP & $21.496 \%$ & $10.474 \%$ \\
& FBMAP & $12.383 \%$ & $3.5199 \%$ \\
\hline \multirow{2}{*}{ LH } & BMAP & $29.370 \%$ & $15.935 \%$ \\
& FBMAP & $13.362 \%$ & $3.3415 \%$ \\
\hline
\end{tabular}

error (ARPE) of the FBMAP is smaller than those of the BMAP in case of ADVANC and LH.

In general, the 10,000 scenarios or sample paths of Brownian motion process are considered. In each path, the ARPE is computed from both models. The comparison results between the BMAP and the FBMAP can be seen from Table 3 . It can be seen that the average ARPE of the FBMAP is less than the average ARPE of the BMAP. Moreover, the standard deviation of ARPE from the FBMAP is smaller compared with the BMAP. The simulation results show that the FBMAP 


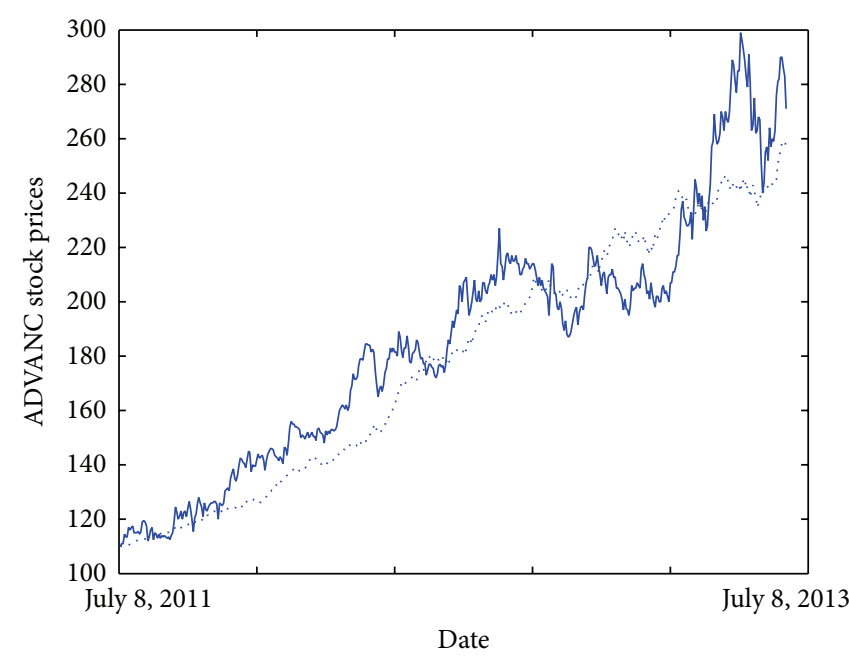

ADVANC empirical prices ADVANC prices simulated by FBMAP

(a) For ADVANC closed prices $(\mathrm{ARPE}=6.9208 \%)$

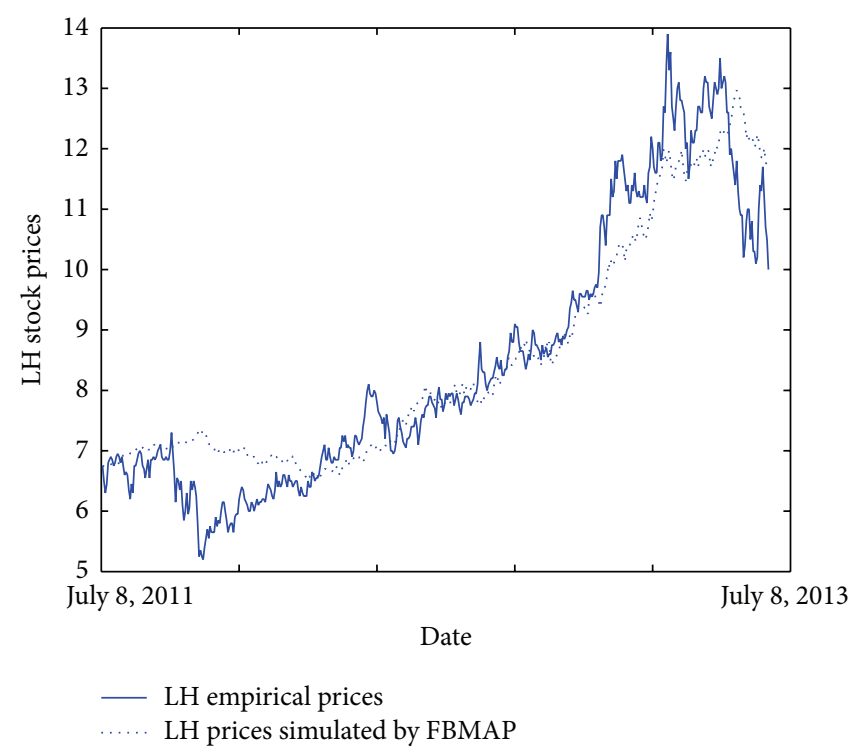

(b) For LH closed prices (ARPE $=6.8788 \%)$

FIGURE 5: The simulation results using the FBMAP.

can provide the small ARPE compared with the BMAP in case of ADVANC and LH.

\section{Conclusion}

Two asset pricing models are presented in the paper. One is the Brownian motion model with adaptive parameters called BMAP and another one is the fractional Brownian motion model with adaptive parameters called FBMAP. The rate of return and volatility in both models are adaptive at any time. The driven process in the BMAP is Brownian motion, while the driven process in the FBMAP is a fractional Brownian motion. The BMAP and the FBMAP are applied to simulate the ADVANC and LH stock prices. The simulated prices from both models are compared with the empirical prices. The accuracy index ARPE is used in the paper. From the 10,000 scenarios of simulated prices of each model, the average and standard deviation of ARPE from both models show that the FBMAP provides a better appropriateness with the dataset than the BMAP in case of ADVANC and LH. Therefore, the FBMAP is suitable to predict the ADVANC and LH closed prices in the future.

\section{Conflict of Interests}

The author declares that there is no conflict of interests regarding the publication of this paper.

\section{References}

[1] F. Black and M. Scholes, "The pricing of options and corporate liabilities," Journal of Political Economy, vol. 81, no. 3, pp. 637654, 1973.
[2] F. Black and M. Scholes, "Taxes and the pricing of options," Journal of Finance, vol. 31, no. 2, pp. 319-332, 1976.

[3] R. C. Merton, "Theory of rational option pricing," The Rand Journal of Economics, vol. 4, pp. 141-183, 1973.

[4] B. B. Mandelbrot and J. W. van Ness, "Fractional Brownian motions, fractional noises and applications," SIAM Review, vol. 10, pp. 422-437, 1968.

[5] E. Alòs, O. Mazet, and D. Nualart, "Stochastic calculus with respect to fractional Brownian motion with Hurst parameter lesser than 1/2," Stochastic Processes and their Applications, vol. 86, no. 1, pp. 121-139, 2000.

[6] T. H. Thao, "An approximate approach to fractional analysis for finance," Nonlinear Analysis: Real World Applications, vol. 7, no. 1, pp. 124-132, 2006.

[7] P. Wilmott, Paul Wilmott on Quantitative Finance, John Wiley \& Sons, Chichester, UK, 2006.

[8] R. Seydel, Tools for Computational Finance, Springer, Berlin, Germany, 2002.

[9] T. H. Thao and T. T. Nguyen, "Fractal Langevin equation," Vietnam Journal of Mathematics, vol. 30, no. 1, pp. 89-96, 2002. 


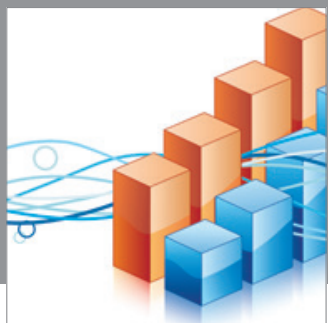

Advances in

Operations Research

mansans

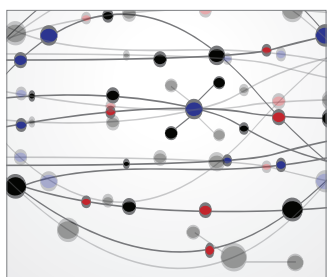

The Scientific World Journal
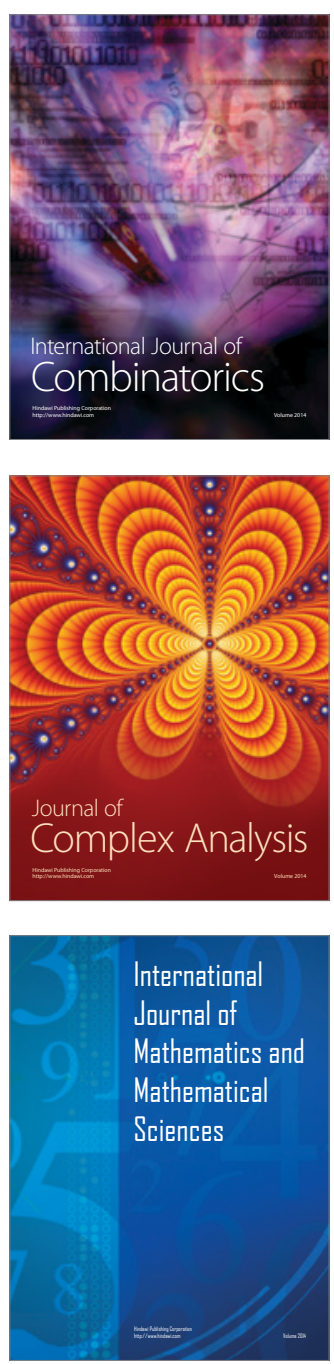
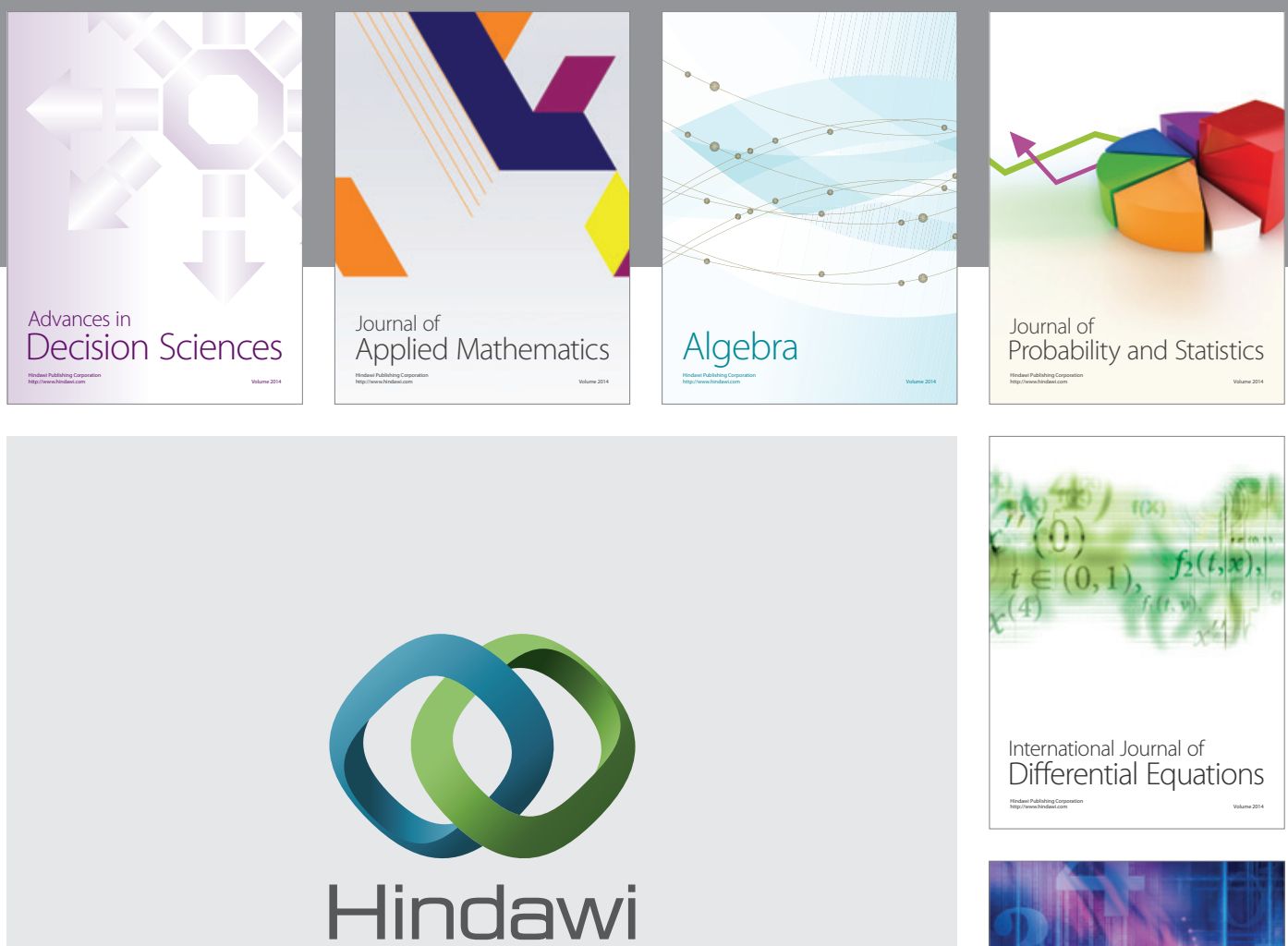

Submit your manuscripts at http://www.hindawi.com
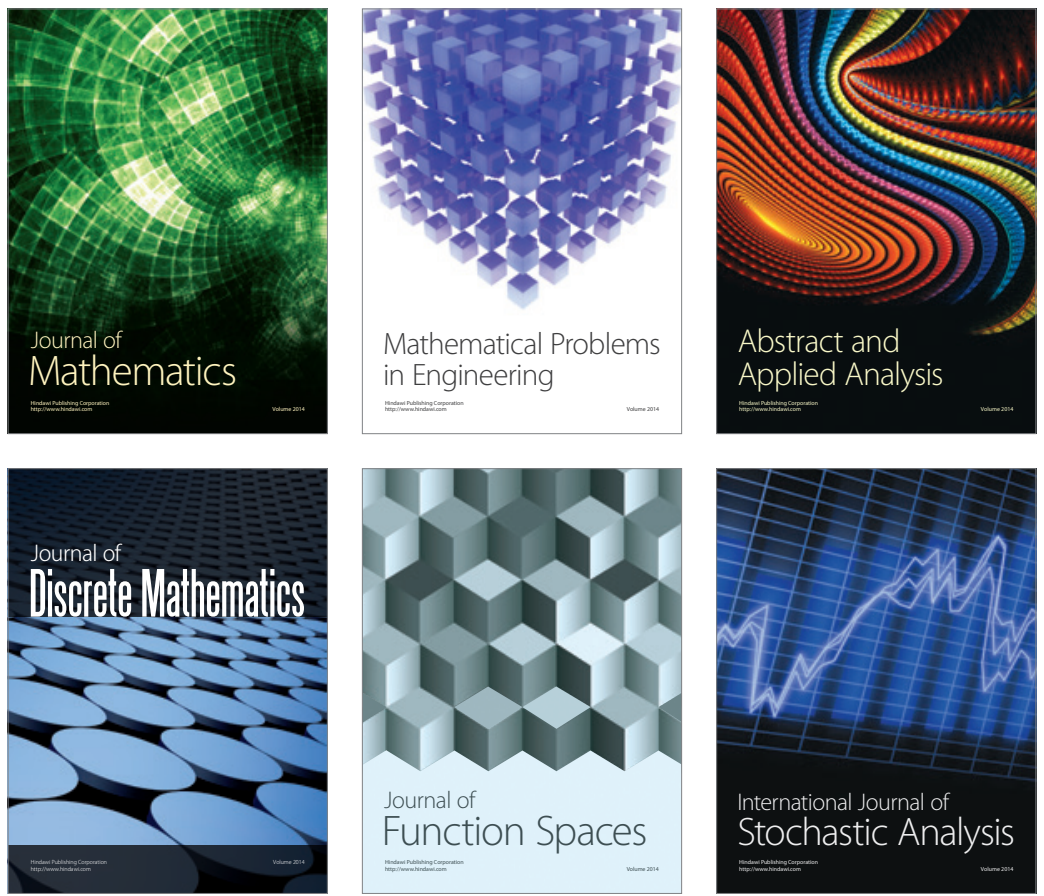

Journal of

Function Spaces

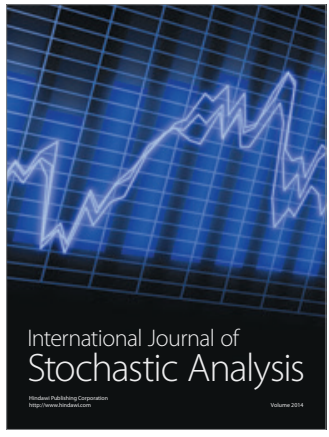

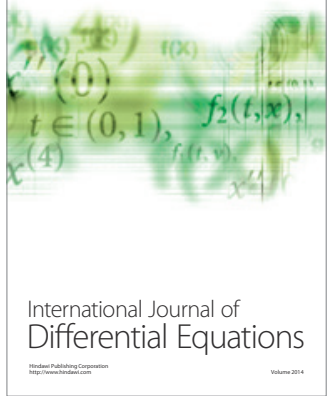
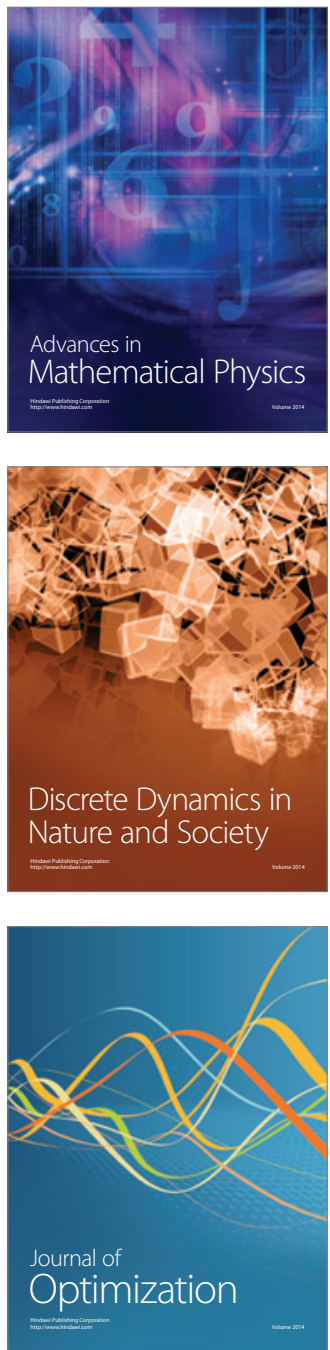